\title{
DNA damage on the DOCK in FLT3-ITD-driven acute myeloid leukemia
}

\author{
Ruchi Pandey and Reuben Kapur \\ Herman B Wells Center for Pediatric Research, Indiana University School of Medicine, IN, USA
}

E-mail: RUCHI PANDEY - pandeyru@iupui.edu or REUBEN KAPUR - rkapur@iupui.edu

doi:10.3324/haematol.2019.231340

I nduction of DNA damage by chemotherapeutics has been the mainstay of cancer therapy irrespective of the origin of the cancer. However, in acute myeloid leukemia (AML) responses to intensive chemotherapy differ greatly, with the success rate ranging very widely from $94 \%$ to $17 \%$ depending upon the karyotype of the patients. ${ }^{1}$ In particular, AML patients with normal cytogenetics initially respond to DNA damaging agents but frequently relapse and have a 5-year survival rate of around $30 \%$. The inferior survival in these patients correlates with the presence of internal tandem duplications (ITD) in FLT3, a cytokine receptor with tyrosine kinase activity, found in almost one-third of AML patients. Constitutively active FLT3-ITD contributes to increased proliferation and survival of myeloid progenitor cells. Although FLT3-ITD by itself is not considered a driver of AML, the presence of the mutation at both diagnosis and relapse highlights the importance of FLT3-ITD in resistance of leukemia-initiating cells to therapy. FLT3-ITD can activate all the major signaling pathways, such as Ras/ERK, JAK/STAT5 and PI3K/AKT but we still do not completely understand how these lead to resistance to chemotherapy and whether they create any vulnerabilities that could be exploited. FLT3-ITD activates the NADPH oxidase system through RAC1 to augment the production of reactive oxygen species (ROS) and a consequent adaptive response involving RAD51-mediated error prone repair and enhanced genomic instability. ${ }^{2}$ Interestingly, increased ROS production is not associated with the tyrosine kinase domain mutated FLT3, which incidentally is also not associated with poor prognosis. These suggest that increased DNA damage response (DDR) and genomic instability are important for the poor response to therapy in FLT3-ITD-positive (FLT3-ITD ${ }^{+}$) AML patients. In the current issue of Haematologica, Wu et al. ${ }^{3}$ provide evidence of involvement of a FLT3ITD/DOCK2/ RAC1 self-sustaining positive feedback loop leading to upregulation of DDR proteins that contributes to chemotherapy resistance (Figure 1). They provide evidence for the crucial role played by DOCK2 in mediating chemoresistance through regulating the RAC1/STAT5/DDR axis. ${ }^{3}$ Although expression of FLT3ITD has been associated with resistance to chemotherapy and it has long been known that inhibition of signaling by kinase inhibitors can sensitize leukemic cells, ${ }^{4}$ this had not been extensively explored or utilized in the clinic. Only recently, is it becoming evident that midostaurin (PKC412), a multi-kinase inhibitor, is much more effective at inducing sustained remissions when used in combination with chemotherapy than when used as singleagent therapy. ${ }^{5}$ The results presented by Wu et al. ${ }^{3}$ further emphasize the role of chemo-sensitization through inhibition of signaling by mutant FLT3 and provide a way for- ward for improving the clinical outcome in FLT3-ITD ${ }^{+}$ AML patients.

Activation of RAC1 by FLT3-ITD has been recognized as the major contributor to enhanced DDR but the ubiquitous expression of RAC1 and its involvement in multiple processes makes targeting RAC1 specifically in leukemic cells a challenge. ${ }^{6}$ DOCK2, an atypical guanine nucleotide exchange factor, has previously been identified as an intermediate between FLT3-ITD and STAT5. DOCK2 has much more restricted expression, acts upstream of RAC1 and is required for upregulation of the DDR pathway. Combining loss of DOCK 2 with cytarabine produced a similar increase in cytotoxicity as that observed with inhibiting the kinase function of FLT3-ITD (Figure 1). ${ }^{3}$ The alternative pathways from FLT3-ITD, such as FAK/TIAM1 and DOCK2, culminating in activation of RAC1 and subsequent nuclear translocation of STAT5 provide additional points of vulnerability that could be therapeutically exploited to overcome acquired drug resistance in response to FLT3-specific small molecule inhibitors. ${ }^{7,8}$ DOCK2 mutations leading to activation of RAC1 have also been identified in gastrointestinal and prostate cancers. It remains to be seen if similar deregulation of the DDR pathway is involved in carcinogenesis across different types of cancers.

The synthetic lethality observed with DOCK2 inhibition upon treatment with cytarabine could be attributed to downregulation of FLT3-ITD and the DDR pathway. However, Wu et al. ${ }^{3}$ did not observe a similar synergism when inhibitors of individual components of the DDR such as CHK1 (MK8776) or WEE1 (MK1775) were used in combination with cytarabine. As expected, the concurrent use of inhibitors of CHK1 and WEE1 with DOCK2 inhibition showed marginal improvements since DOCK2 knockdown significantly reduces both FLT3-ITD and these DDR pathway proteins. ${ }^{3}$ Functional redundancy between DDR proteins may account for lack of synergism between MK8776 or MK1775 and cytarabine. Although targeting DOCK2 appears to be more beneficial; the clinical translation of co-targeting DOCK2 is limited due to non-availability of potent and specific inhibitors. Currently two small molecule inhibitors of DOCK2 are available: the chemically synthesized 4-[3'(2"-chlorophenyl)-2'-propen-1'-ylidene]-1-phenyl-3,5pyrazolidinedione (CPYPP) ${ }^{9}$ and a naturally occurring cholesterol sulfate. ${ }^{10}$ The effects of these inhibitors of DOCK2 have been evaluated in the immune system and they have been found to block RAC1 activation but a comprehensive evaluation in AML models has not been carried out. The significance of targeting DOCK2 in sensitizing FLT3-ITD ${ }^{+}$AML cells to chemotherapy demonstrated by Wu et al. ${ }^{3}$ may provide the impetus for development of therapeutic strategies to target DOCK2 in 


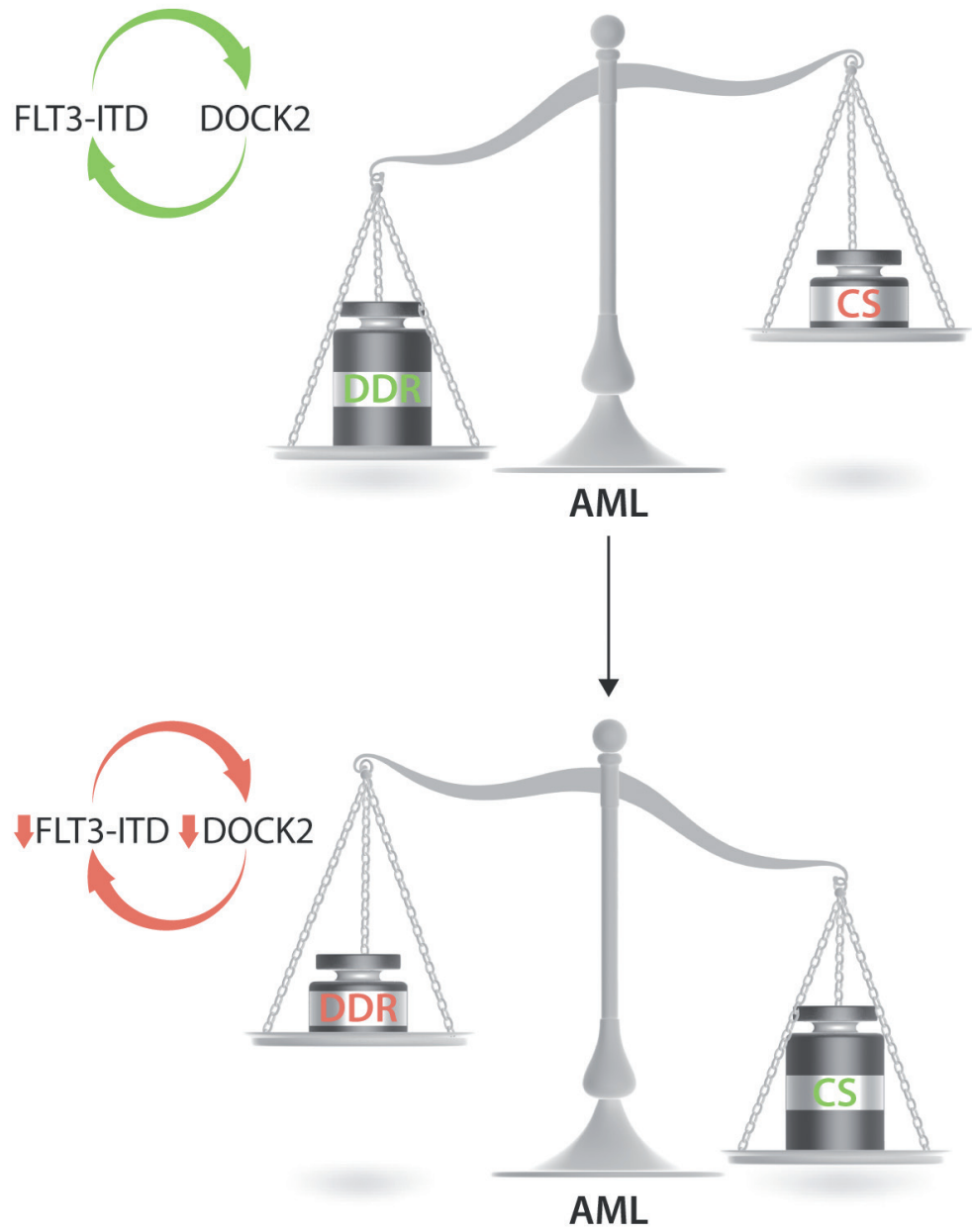

Figure 1. FLT3-ITD and DOCK2 maintain the balance between the DNA damage response and chemosensitivity. The positive signaling feedback between FLT3-ITD and DOCK2 upregulates the DNA damage response (DDR) through RAC1 leading to a decrease in chemosensitivity (CS) of acute myeloid leukemia (AML) cells. The balance can be tilted in favor of chemotherapy by downregulation of DOCK2 or inhibition of FLT3-ITD or DDR proteins which sensitizes the AML cells.

combination with standard chemotherapy leading to better prognosis for these AML patients.

While the study by $\mathrm{Wu}$ et $a .^{3}{ }^{3}$ focuses only on FLT3$\mathrm{ITD}^{+} \mathrm{AML}$, similar defects in the DDR are known to be associated with several other AML driver mutations, specifically the epigenetic regulators. Genes such as DNMT3A and TET2, which are very frequently mutated not only in the leukemic cells but also in the preleukemic clones, are known to regulate the DDR. ${ }^{11,12}$ Cells carrying mutations in these genes are known to have an increased propensity to accumulate DNA damage. Interestingly, they are also found in non-random association with FLT3-ITD and their concurrent occurrence is sufficient for AML initiation. Similar to FLT3ITD these pre-leukemic mutant clones tend to be chemoresistant and they expand following elimination of leukemic cells by chemotherapy. The pre-leukemic clones are believed to evolve into leukemic cells due to inherent genomic instability and acquisition of additional mutations. The clinical relevance of alterations in the DDR in AML due to genetic mutations is underscored by the increase in survival with induction therapy dose escalation observed in patients carrying mutant DNMT3A but not in their wildtype counterparts. ${ }^{13}$ With better understanding of the driver mutations in AML and their role in normal cell functions, it is becoming appar- ent that an altered DDR is a common underlying theme in AML and pre-leukemic mutant cells. The research by $\mathrm{Wu}$ et al. ${ }^{3}$ emphasizes that combinatorial treatment strategies tailored to the specific driver mutation-dependent vulnerability in the DDR need to be explored further. A similar approach of inducing synthetic lethality by using PARP inhibitors in combination with standard chemotherapy in BRCA mutated gynecological cancers has led to improved patients' outcomes in the clinic. ${ }^{14}$ Interestingly, FLT3-ITD ${ }^{+}$leukemic cells can be sensitized to PARP inhibitors when used in combination with AC220, a second-generation FLT3 inhibitor, leading to enhanced survival. ${ }^{15}$ It is to be hoped that these studies will spur development of rational combinations of inhibitors of FLT3 signaling with DDR inhibitors leading to a broader range of options to improve patients' outcomes and survival.

\section{Acknowledgments}

$R P$ is supported by an award from the Ralph W. and Grace M. Showalter Research Trust and Indiana University School of Medicine. $R K$ is supported by research grants from NIHR01CA173852, R01CA134777, R01HL146137, and R01HL140961 and the Riley Children's Foundation. The content of this editorial is solely the responsibility of the authors and does not represent the views of the funding agencies. 


\section{References}

1. Byrd JC, Mrozek K, Dodge RK, et al. Cancer, Leukemia Group B. Pretreatment cytogenetic abnormalities are predictive of induction success, cumulative incidence of relapse, and overall survival in adult patients with de novo acute myeloid leukemia: results from Cancer and Leukemia Group B (CALGB 8461). Blood. 2002;100(13):43254336.

2. Sallmyr A, Fan J, Datta K, et al. Internal tandem duplication of FLT3 (FLT3/ITD) induces increased ROS production, DNA damage, and misrepair: implications for poor prognosis in AML. Blood. 2008;111(6):3173-3182.

3. Wu M, Li L, Hamaker M, Small D, Duffield AS. FLT3-ITD cooperates with Rac1 to modulate the sensitivity of leukemic cells to chemotherapeutic agents via regulation of DNA repair pathways. Haematologica. 2019;104(12):2418-2428.

4. Seedhouse CH, Hunter HM, Lloyd-Lewis B, et al. DNA repair contributes to the drug-resistant phenotype of primary acute myeloid leukaemia cells with FLT3 internal tandem duplications and is reversed by the FLT3 inhibitor PKC412. Leukemia. 2006;20(12): 2130-2136.

5. Stone RM, Mandrekar SJ, Sanford BL, et al. Midostaurin plus chemotherapy for acute myeloid leukemia with a FLT3 mutation. N Engl J Med. 2017;377(5):454-464.

6. Marei H, Malliri A. Rac1 in human diseases: the therapeutic potential of targeting Rac1 signaling regulatory mechanisms. Small GTPases. 2017;8(3):139-163.
7. Wu M, Hamaker M, Li L, Small D, Duffield AS. DOCK2 interacts with FLT3 and modulates the survival of FLT3-expressing leukemia cells. Leukemia. 2017;31(3):688-696

8. Chatterjee A, Ghosh J, Ramdas B, et al. Regulation of Stat5 by FAK and PAK1 in oncogenic FLT3- and KIT-driven leukemogenesis. Cell Rep. 2014;9(4):1333-1348.

9. Nishikimi A, Uruno T, Duan X, et al. Blockade of inflammatory responses by a small-molecule inhibitor of the Rac activator DOCK2. Chem Biol. 2012;19(4):488-97.

10. Sakurai T, Uruno T, Sugiura Y, et al. Cholesterol sulfate is a DOCK2 inhibitor that mediates tissue-specific immune evasion in the eye. Sci Signal. 2018;11(541)pii:eaao4874.

11. Guryanova OA, Shank K, Spitzer B, et al. DNMT3A mutations promote anthracycline resistance in acute myeloid leukemia via impaired nucleosome remodeling. Nat Med. 2016;22(12):1488-1495.

12. Pan F, Wingo TS, Zhao Z, et al. Tet2 loss leads to hypermutagenicity in haematopoietic stem/progenitor cells. Nat Commun. 2017;8:15102.

13. Sehgal AR, Gimotty PA, Zhao J, et al. DNMT3A mutational status affects the results of dose-escalated induction therapy in acute myelogenous leukemia. Clin Cancer Res. 2015;21(7):1614-1620.

14. Lord CJ, Ashworth A. PARP inhibitors: synthetic lethality in the clinic. Science. 2017;355(6330):1152-1158.

15. Maifrede S, Nieborowska-Skorska M, Sullivan-Reed K, et al Tyrosine kinase inhibitor-induced defects in DNA repair sensitize FLT3(ITD)-positive leukemia cells to PARP1 inhibitors. Blood. 2018;132(1):67-77

\title{
Fine tuning of $\mathrm{p} 53$ functions between normal and leukemic cells: a new strategy for the treatment of chronic lymphocytic leukemia
}

\author{
Tatjana Stankovic
}

Institute of Cancer and Genomic Sciences, University of Birmingham, Birmingham, UK

E-mail: TATJANA STANKOVIC - t.stankovic@bham.ac.uk

doi:10.3324/haematol.2019.230896

\section{Pathophysiology and current therapies in chronic lymphocytic leukemia}

Chronic lymphocytic leukemia (CLL) is the most frequent leukemia in western countries. It is characterized by the accumulation of mature B lymphocytes in the peripheral blood, peripheral lymphoid organs and bone marrow. CLL displays a heterogeneous clinical course, ranging from protracted indolent disease with no requirement for treatment in some patients to rapid disease progression and subsequent treatment refractoriness in others. ${ }^{1-3}$

CLL progression is a reflection of the complex interplay between genomic drivers of disease and interactions with the microenvironment. ${ }^{4}$ Whole genome/exome profiling by next-generation sequencing has revealed that the clonal composition of CLL is constantly reshaped during disease progression. It has been proposed that CLL exhibits a stochastic model of progression with the existence of a 'trunk' tumor population and numerous 'branches' that can act as tumor progenitors. According to this model, the subclonal topography of CLL arises over time as a result of an initial driver mutation which leads to malignant transformation and is observed in all tumor cells the trunk population. This is followed by secondary driver mutations in distinct subclones which are selected by intraclonal competition or treatment, and are likely to contribute to disease progression. Finally, CLL relapse has been attributed to the expansion of highly fit, often treatment-selected subclones (branches) carrying mutations in the DNA damage response (DDR) genes TP53 and ATM, SF3B1 or NRAS., As a result, a significant proportion of relapsed/refractory CLL can be attributed to the functional loss of the DDR.

For several decades, alkylating agents and purine analogs were the principal therapies for CLL, augmented by the addition of monoclonal antibodies. The last decade has seen an expansion in the number of compounds targeting specific aspects of the CLL phenotype, from the interactions of tumor cells with the microenvironment and B-cell receptor signaling to anti-apoptotic cellular pathways, heralding a new era of CLL therapy based on targeted treatment approaches. ${ }^{7-10}$ In particular, new inhibitors of signaling pathways that are critical to CLL survival and proliferation, such as Bruton tyrosine kinase (BTK), phosphoinositide-3 kinase (PI3K), and the anti-apoptotic protein $\mathrm{Bcl}-2$, have changed the management of many CLL patients.

Despite the array of available therapeutic options, CLL remains, at present, an incurable condition. ${ }^{11}$ Firstly, the acquisition of DDR gene defects such as TP53 deletions and/or mutations renders CLL patients refractory to conventional chemoimmunotherapies. The clinical response to the BTK inhibitor, ibrutinib, is encouraging for some but not all refractory tumors. ${ }^{12}$ Secondly, clonal selection 